\title{
Umsetzung der schulischen Integration von SchülerInnen mit Behinderung(en) in Österreich
}

\author{
Susanne Schwab, Markus Gebhardt \& Mathias Krammer
}

\section{Schulische Integration in Österreich}

Gemeinsamer Unterricht von SchülerInnen mit und ohne Behinderung(en) findet in Österreich vorwiegend in der Organisationsform von so genannten Integrationsklassen statt. Laut Statistik Austria wird aktuell etwa die Hälfte aller SchülerInnen mit sonderpädagogischen Förderbedarf (kurz: SPF) integrativ unterrichtet (Statistik Austria 2012), wobei die Zahlen zwischen den einzelnen Bundesländern stark schwanken. In der Steiermark werden über $80 \%$ der SchülerInnen mit SPF in Integrationsklassen beschult, während es in Niederösterreich nur knapp 30\% sind (Buchner/Gebhardt 2011). Unabhängig von der Art der Behinderung eines Kindes können Eltern bzw. Erziehungsberechtigte sich entweder für eine integrative oder segregative Schulform entscheiden. Förderungen und Ressourcen können nur SchülerInnen mit einem diagnostizierten Förderbedarf beanspruchen. Dieser wird in Österreich im Auftrag der Schulaufsicht von SonderpädagogInnen geprüft. Auf der Basis der erstellen sonderpädagogischen Gutachten wird ein SPF per Bescheid zuerkannt oder abgelehnt. Anschließend können Lehrplaneinstufungen nach den im Schulorganisationsgesetz verankerten Varianten (z.B. Lehrplan der Sonderschule für Gehörlose, Lehrplan der Sondererziehungsschule u.a.) vorgenommen werden. Eine standardisierte Vorgehensweise für die Gutachtenerstellung gibt es jedoch nicht (kritisch hierzu z.B. Schwab 2014).

Die Umsetzung der Integration obliegt den einzelnen Bundesländern (Buchner/Gebhardt 2011). Grundsätzlich werden Integrationsklassen erst ab mindestens drei SchülerInnen mit SPF gebildet. Sie müssen eine kleinere Klassengrößen sowie ein Zwei-LehrerInnensystem aufweisen. Bei nur einzelnen SchülerInnen mit SPF wird die zweite Lehrperson lediglich für einzelne Stunden eingesetzt. Die Ressourcenbindung an Etikettierungen ist problematisch, denn sie kann zu Stigmatisierungen führen.

Die aktuell geltenden organisatorischen Rahmenbedingungen ermöglichen es einen gemeinsamen Unterricht umzusetzen. Um von der schulischen Integration zu einer schulischen Inklusion zu gelangen benötigt es aber auch eine spezi- 
fische Unterrichtsentwicklung, die auf eine Didaktik der Vielfalt abzielt. Die schulischen Bedingungen müssen so adaptiert werden, dass bei der Förderung alle SchülerInnen mit ihren individuellen Bedürfnissen erreicht werden. Effektive Co-Teaching-Modelle (Schwab/Holzinger/Krammer/Gebhardt/Hessels eingereicht) und auch andere Lernformen (z.B. Arbeiten mit einem Wochenplan, Stationslernen, Freiarbeit und Gesprächskreise; siehe dazu auch Heimlich 2007) sind das Ziel einer inklusiven LehrerInnenausbildung. Inwieweit dies in der Praxis auch umgesetzt wird, ist aktuell noch offen.

\section{Ziele und Fragestellungen}

Im Rahmen des vorliegenden Beitrags wird untersucht, wie die schulische Integration in den steirischen Integrationsklassen aktuell umgesetzt wird. Die leitenden Fragestellungen sind hierbei:

1. In welchen Bereichen unterstützen die sonderpädagogischen Zentren (kurz: SPZ) SonderpädagogInnen und RegelschullehrerInnen?

2. Wie häufig finden Partnerarbeit, Gruppenarbeit, Wochenplanarbeit oder andere offene Unterrichtsformen im Unterricht statt?

3. Sind SonderpädagogInnen und RegelschullehrerInnen mit der Zusammenarbeit im LehrerInnenteam zufrieden?

Da vermutet werden kann, dass sich zwischen Integrationsklassen in Primarstufen (welche eine/n fixe/n KlassenlehrerIn haben) und jenen in der Sekundarstufe (mit FachlehrerInnensystem) große Unterschiede ergeben können (Gebhardt/Krammer/Schwab/Gasteiger-Klicpera 2013), werden die genannten Forschungsfragen jeweils getrennt für diese beiden Gruppen beantwortet.

\section{Empirische Untersuchung}

\subsection{Vorgehensweise und Beschreibung der Stichprobe}

An der Befragung nahmen insgesamt 340 LehrerInnen teil welche in steirischen Integrationsklassen (aller Schulbezirke in der Steiermark) arbeiten. Die Befragung erfolgte postalisch, wobei die LeiterInnen der SPZ vorab persönlich über die Studie informiert wurden und diese die Fragebögen persönlich an LehrerInnen aus Integrationsklassen verteilten. Dabei fand gemeinsam eine Stichprobenauswahl (nach Schulgröße und Schulbezirk) statt, um eine repräsentative Stichprobe zu erhalten. Aus datenschutzrechtlichen Gründen war es nicht möglich, Schulen direkt zu kontaktieren. Die LehrerInnen schickten die anonymen Frage- 
bögen direkt an die Universität. Die Rücklaufquote kann insgesamt auf über $80 \%$ geschätzt werden (siehe dazu z.B. auch Gebhardt et al. 2013). Von den teilnehmenden LehrerInnen waren 212 RegelschullehrerInnen (162 Frauen, 42 Männer) und 128 waren SonderschullehrerInnen (120 Frauen, 8 Männer). Das Alter der TeilnehmerInnen belief sich auf durchschnittlich 46.5 Jahre (SD=9.4) und die Lehrerfahrung war im Durchschnitt 21.9 Jahre (SD=11.5) im Regelunterricht beziehungsweise 9.7 Jahre $(\mathrm{SD}=6.3)$ im integrativen Unterricht.

\subsection{Befragungsinstrument}

Der verwendete Fragebogen wurde in Anlehnung an eine Voruntersuchung von Holzinger, Ebner, Kernbichler, Kopp-Sixt, Much und Pongratz (2011) entwickelt. Beschreibungen des Instruments finden sich auch unter Gebhardt et al. (2013) sowie bei Gebhardt, Schwab, Krammer und Gegenfurtner (eingereicht) sowie bei Schwab et al. (eingereicht). Im Folgenden werden lediglich die für die vorliegenden Analysen relevanten Items genauer beschrieben. Der Frageblock, welcher die Unterstützung des SPZ erfasst, enthält insgesamt vier Items (Individuelle Beratung, Informationszentrum und Austausch, Angebote für Fortbildungen und Veranstaltungen, Gutachtenerstellung und Diagnostik), welche auf einer fünfstufigen Likertskala von sehr wenig bis sehr viel erfasst wurden (siehe Tabelle 1). Zudem wurde gebeten, weitere Unterstützungsleistungen beschreibend anzugeben. Bei den offenen Unterrichtsformen wurden drei Formen vorgegeben (Partnerarbeit, Gruppenarbeit und Wochenplanarbeit) und zudem noch die Möglichkeit eröffnet, anzugeben, wie häufig andere offene Unterrichtsformen angewendet werden. Das Antwortformat war fünfstufig (nie - selten - 1x pro Woche - 2-3x pro Woche - täglich). Die Frage der Beurteilung der Zusammenarbeit im Team wurde so formuliert, dass auf einer siebenstufigen Likertskala (mangelhaft - optimal) die Zusammenarbeit für das komplette LehrerInnenteam einer Klasse beurteilt wird.

\subsection{Ergebnisse}

Tabelle 1 sind die Einschätzungen über die Unterstützungsleistungen der SPZ in den einzelnen Bereichen zu entnehmen. Vergleicht man die dort eingetragenen Mittelwerte mit dem theoretischen Skalenmittelwert von 3 (bei einer fünfstufigen Skala von 1 -5), so kann man erkennen, dass für die Primarstufe alle Mittelwerte über dem theoretischen Skalenmittelwert liegen, jene der Sekundarstufe sind hingehen oftmals etwas niedriger. Alle vier Gruppen (RegelschullehrerInnen sowie SonderschullehrerInnen der Primarstufe sowie der Sekundarstufe) sehen 
die größte Unterstützungsleistung dabei im Bereich der Gutachtenerstellung und Diagnostik. Insgesamt wurden lediglich viermal sonstige Unterstützungsleistungen angegeben („Motopädadagogik und LRS-Spezialistin“; „Unterstützung in besonderen Fällen“; „Unterstützung mit Materialien“; „Hilfe durch "Fachleute").

Tabelle 1: Angaben über die Unterstützungsleistungen der SPZ

\begin{tabular}{ccccc}
\hline & $\begin{array}{c}\text { Regelschul- } \\
\text { lehrerInnen der } \\
\text { Primarstufe } \\
\mathrm{M}(\mathrm{SD})\end{array}$ & $\begin{array}{c}\text { Sonderschul- } \\
\text { lehrerInnen der } \\
\text { Primarstufe } \\
\mathrm{M}(\mathrm{SD})\end{array}$ & $\begin{array}{c}\text { Regelschul- } \\
\text { lehrerInnen der } \\
\text { Sekundarstufe } \\
\mathrm{M}(\mathrm{SD})\end{array}$ & $\begin{array}{c}\text { Sonderschul- } \\
\text { lehrerInnen der } \\
\text { Sekundarstufe } \\
\text { M (SD) }\end{array}$ \\
\hline $\begin{array}{c}\text { Individuelle } \\
\text { Beratung } \\
\text { Informations- } \\
\text { zentrum und } \\
\text { Austausch }\end{array}$ & $3.60(1.51)$ & $3.33(1.42)$ & $2.86(1.31)$ & $2.89(1.40)$ \\
$\begin{array}{c}\text { Angebote für } \\
\text { Fortbildungen } \\
\text { und Veranstal- } \\
\text { tungen }\end{array}$ & $3.45(1.42)$ & $3.25(1.36)$ & $2.93(1.31)$ & $2.81(1.23)$ \\
$\begin{array}{c}\text { Gutachten- } \\
\text { erstellung und } \\
\text { Diagnostik }\end{array}$ & $4.42(0.99)$ & $3.31(1.04)$ & $3.04(1.17)$ & $3.15(1.25)$ \\
\hline Range: Min =1, Max $=5$ & $4.17(0.91)$ & $3.27(1.26)$ & $2.68(1.36)$ \\
\hline
\end{tabular}

Bezüglich der Umsetzung von offenen Unterrichtsformen (siehe Tabelle 2) zeigt sich, dass die Mehrheit der LehrerInnen entweder einmal oder zwei bis dreimal pro Woche offene Unterrichtsformen anwenden. Partnerarbeit wird insgesamt bei allen LehrerInnengruppen am häufigsten angewendet. Auch gaben etwa $10 \%$ der LehrerInnen an, dass sie nie mit Wochenplänen arbeiten. 
Tabelle 2: Umsetzung von offenen Unterrichtsformen

\begin{tabular}{lccccc}
\hline & nie & selten & 1x/Woche & $2-3 \times /$ Woche & täglich \\
\hline RegelschullehrerInnen der Primarstufe & & & & & \\
Partnerarbeit & $0 \%$ & $6.3 \%$ & $12.7 \%$ & $58.7 \%$ & $22.2 \%$ \\
Gruppenarbeit & $0 \%$ & $29 \%$ & $29 \%$ & $35.5 \%$ & $6.5 \%$ \\
Wochenplanarbeit & $11.1 \%$ & $27 \%$ & $38.1 \%$ & $22.2 \%$ & $1.6 \%$ \\
Andere offene Unterrichtsformen & $1.7 \%$ & $11.9 \%$ & $27.1 \%$ & $39 \%$ & $20.3 \%$ \\
\hline SonderschullehrerInnen der Primarstufe & & & & \\
Partnerarbeit & $1.6 \%$ & $9.8 \%$ & $18 \%$ & $50.8 \%$ & $19.7 \%$ \\
Gruppenarbeit & $0 \%$ & $24.6 \%$ & $31.1 \%$ & $39.3 \%$ & $4.9 \%$ \\
Wochenplanarbeit & $9.7 \%$ & $32.3 \%$ & $27.4 \%$ & $22.6 \%$ & $8.1 \%$ \\
Andere offene Unterrichtsformen & $1.9 \%$ & $14.8 \%$ & $33.3 \%$ & $33.3 \%$ & $16.7 \%$ \\
\hline RegelschullehrerInnen der Sekundarstufe & & & & \\
Partnerarbeit & $0.9 \%$ & $5.4 \%$ & $34.2 \%$ & $43.2 \%$ & $16.2 \%$ \\
Gruppenarbeit & $1.9 \%$ & $23.1 \%$ & $41.7 \%$ & $25 \%$ & $8.3 \%$ \\
Wochenplanarbeit & $8.4 \%$ & $53.3 \%$ & $18.7 \%$ & $10.3 \%$ & $9.3 \%$ \\
Andere offene Unterrichtsformen & $2.9 \%$ & $32.4 \%$ & $34.3 \%$ & $16.7 \%$ & $13.7 \%$ \\
\hline SonderschullehrerInnen der Sekundarstufe & \multicolumn{3}{c}{} \\
Partnerarbeit & $0 \%$ & $3.2 \%$ & $23.8 \%$ & $36.5 \%$ & $36.5 \%$ \\
Gruppenarbeit & $1.6 \%$ & $9.5 \%$ & $46 \%$ & $34.9 \%$ & $7.9 \%$ \\
Wochenplanarbeit & $9.7 \%$ & $22.6 \%$ & $22.6 \%$ & $19.4 \%$ & $25.8 \%$ \\
Andere offene Unterrichtsformen & $1.7 \%$ & $21.7 \%$ & $38.3 \%$ & $25 \%$ & $13.3 \%$ \\
\hline
\end{tabular}

In Bezug auf die Bewertung der Teamzusammenarbeit gaben die LehrerInnen insgesamt an, dass sie sehr zufrieden sind. Der Mittelwert der Gesamtstichprobe lag bei $6.12(\mathrm{SD}=1.21)$ und ist auf der siebenstufigen Ratingskala (1-7) als hoch einzustufen. Mittels Varianzanalyse wurde geprüft, ob sich diesbezüglich Unterschiede zwischen den vier LehrerInnengruppen zeigen. Die Ergebnisse bestätigen das Vorliegen von Gruppenunterschieden $(\mathrm{F}[2,288]=9.4, \mathrm{p}<.01$, Eta2=.09). Post-Hoc Vergleiche nach Scheffé zeigten keine Unterschiede in der Einschätzung der Teamzusammenarbeit zwischen KlassenlehrerInnen $(\mathrm{M}=6.55$, $\mathrm{SD}=0.85)$ und SonderschullehrerInnen der Primarstufe $(\mathrm{M}=6.44, \mathrm{SD}=0.88)$. Auch innerhalb der Sekundarstufe gab es keine signifikanten Unterschiede im Rating der KlassenlehrerInnen $(\mathrm{M}=5.65, \mathrm{SD}=1.32)$ und der SonderschullehrerInnen $(\mathrm{M}=6.11, \mathrm{SD}=1.16)$. KlassenlehrerInnen der Primarstufe schätzen jedoch die Zusammenarbeit besser ein als LehrerInnen der Sekundarstufe und die Angaben der IntegrationslehrerInnen der Primarstufe waren signifikant positiver als jene der IntegrationslehrerInnen der Sekundarstufe. 


\section{Diskussion}

Eine gelingende inklusive Beschulung setzt neben der schulischen Integration beziehungsweise dem reinen Placement von SchülerInnen mit sonderpädagogischem Förderbedarf (SPF) in Regelklassen auch eine gute Zusammenarbeit mit den Sonderpädagogischen Zentren (SPZ), eine inklusive Didaktik sowie eine funktionierende Teamarbeit voraus. In einer postalischen Erhebung wurden Regel- und SonderschullehrerInnen der Primar- und Sekundarstufe über diese genannten Punkte befragt. Anhand der Ergebnisse lässt sich erkennen, dass die SPZs insbesondere für die Primarstufe eine große Unterstützung leisten, welche im Bereich der Gutachtenerstellung und Diagnostik am stärksten wahrgenommen wird. Da die Diagnose des SPF sowie auch die Lehrplaneinstufung zumeist in den ersten Schuljahren vorgenommen werden, scheint dies nicht weiter verwunderlich. Mit Blick auf die Umsetzung von offenen Unterrichtsformen lässt sich erkennen, dass diese noch nicht an der Tagesordnung stehen, sondern eher ein bis dreimal pro Woche in den Unterricht eingebaut werden. Insbesondere die Wochenplanarbeit wird von etwa zehn Prozent der TeilnehmerInnen komplett abgelehnt.

Die Einschätzung der Teamzusammenarbeit wird als nahezu optimal gesehen. Insgesamt sind PrimarstufenlehrerInnen zufriedener als LehrerInnen der Sekundarstufe. Dies ist vermutlich darauf zurückzuführen, dass in der Primarstufe ein kleines festes LehrerInnenteam besteht, während in der Sekundarstufe das FachlehrerInnensystem vorherrscht. Insofern sind auch die Teams größer und es ist schwieriger einen informellen Austausch zu organisieren. Eine Limitation der Studie ist es, dass die Zusammenarbeit der LehrerInnen von den LehrerInnen selbst beurteilt wurde. Zwar wurde insgesamt nach der Leistung des gesamten Teams gefragt, da die LehrerInnen aber auch selbst ein Teil des Teams sind, kann hier eine positive Überschätzung vermutet werden. Beobachtungsverfahren hätten diese Mängel nicht, sind jedoch deutlich aufwändiger und kostenintensiver. Die vorliegende Studie kann somit nur einen kleinen Beitrag zur Klärung der Fragestellung liefern. So kann man zusammenfassend sagen, dass Ziele und Forderungen Inklusiver Pädagogik in Bezug auf Schülerinnen und Schüler mit Behinderung(en) in der Steiermark umgesetzt werden und den LehrerInnen bekannt sind. Auch sind wichtige Rahmenbedingungen vorhanden. Aktuelle Herausforderungen der Inklusion liegen eher im Detail der Umsetzung der schulischen Förderung und der sozialen Partizipation der SchülerInnen mit SPF im Unterricht. Engere Wirkfaktoren kann aber erst eine Beobachtungsstudie klären. 


\section{Literatur}

Bm:ukk (Hrsg.) (2009): Sonderpädagogik aus inklusiver Sicht. Wien: Jugend \& Volk. Buchner, Tobias/Gebhardt, Markus: Zur schulischen Integration in Österreich (2011): Historische Entwicklung, Forschung und Status Quo. Zeitschrift für Heilpädagogik. 62. 298-304.

Gebhardt, Markus/Krammer, Mathias/Schwab, Susanne/Gasteiger-Klicpera, Barbara (2013): Zu-sammenarbeit zwischen KlassenlehrerIn und SonderpädagogIn in der Integration. Eine Untersuchung in integrativen Klassen in der Steiermark. Heilpädagogische Forschung. 2. 52-61.

Gebhardt, Markus/Schwab, Susanne/Krammer, Mathias/Gegenfurtner, Andreas (eingereicht): General and Special Education Teachers' Perceptions of Teamwork at Primary and Secondary School Levels. A quantitative survey of Styria teachers.

Heimlich, Ulrich (2007): Didaktik des gemeinsamen Unterrichts. In: Walter, Jürgen/ Wember, Franz (2007): 357-375.

Holzinger, Andrea/Ebner, Manuela/Kernbichler, Gerda/Kopp-Sixt, Silvia/Much, Peter/Pongratz, Hanns Jörg (2011): Forschungsprojekt Qualität in der Integration: Projektzeitraum WS 2008/09 - WS 2010/11. Graz: PH Steiermark.

Schwab, Susanne (im Druck): Achievement Goals in Students with Learning Disabilities, Emotional or Behavioral Disorders and Low-IQ Students without Special Educational Needs. Journal of Cognitive Education on Psychology.

Schwab, Susanne (2014): Schulische Integration, soziale Partizipation und emotionales Wohlbefinden in der Schule. Ergebnisse einer empirischen Längsschnittstudie. Wien: Lit Verlag.

Schwab, Susanne/Holzinger, Andrea/Krammer, Mathias/Gebhardt, Markus/Hessels, Marco G. P. (eingereicht): Looking beyond teacher work and examining needs for inclusive education.

Statistik Austria (2012): Schülerinnen und Schüler mit sonderpädagogischem Förderbedarf 2011/12. Verfügbar unter: http://www.statistik.at/web_de/statistiken/bildung und_kultur/formales_bildungswesen/schulen_schulbesuch/index.html [26.10.2013].

Walter, Jürgen/Wember, Franz B. (Hrsg.) (2007): Sonderpädagogik des Lernens. Handbuch Sonderpädagogik Band 2. Bern: Hogrefe. 